\title{
SOHO/SUMER Results: Mass Flows
}

\author{
Hardi Peter \\ Kiepenheuer-Institut für Sonnenphysik, \\ Schöneckstr. 6, 79104 Freiburg, Germany \\ email: peter@kis.uni-freiburg.de
}

\begin{abstract}
Hardly any part of the chromosphere and the low corona of the Sun is in a static state. Spectrographs reveal line shifts and nonthermal broadening indicating mass supply to the corona, draining of coronal material, syphon flows, wave propagation and more. Imaging instruments show apparent motions of bright or dark structures indicating the presence of flows and waves, too.

This paper will review recent observations of the EUV spectrometer SUMER onboard SOHO on Doppler shifts of lines from the transition region and low corona of the Sun. These results will be discussed with respect to the general picture(s) we have of the structure of the upper solar atmosphere.
\end{abstract}

\section{Introduction}

The mass balance of the upper solar atmosphere is still a puzzling problem. Since the mid 1970ies we know of persistent redshifts of emission lines from the middle of the transition region from the chromosphere to the corona (Doshek et al. 1976). During that period it was also discovered that coronal holes are the source of the fast solar wind (Krieger et al. 1973) and that emission lines formed at coronal temperatures are blueshifted in coronal holes (Rottman et al. 1981). It was not until recently that it became clear that these coronal lines are also blueshifted in regions of the quiet Sun (Peter 1999a, Peter \& Judge 1999).

The latter result as well as numerous studies on flows in individual loops, active region systems and various quiet Sun structures would not have been achieved without the SOHO mission (Solar and Heliospheric Observatory; Fleck et al. 1995) and its EUV spectrometer SUMER (Solar Ultraviolet Measurements of Emitted Radiation; Wilhelm et al. 1995, 1997; Lemaire et al. 1997).

SUMER was specifically designed to study the transition region and upper chromosphere by means of EUV emission lines and EUV continua from $\sim 770$ to $\sim 1600 \AA$ (first order). Thus SUMER nicely covers temperatures from the temperature minimum in the chromosphere $(<5000 \mathrm{~K}$; EUV continua) well into the corona (see Fig. 1 for some lines and their formation temperature). With a spatial resolution of $1-2^{\prime \prime}$ the spectral pixel size of the SUMER detector is $\sim 44 \mathrm{~m} \AA$ corresponding to $\sim 10 \mathrm{~km} / \mathrm{s}$ at $1400 \AA$. The position of an emission line can be determined with sub pixel resolution, however, and in an extensive study Peter \& Judge (1999) have shown that typically the position of a Gaussian 
fit to an emission line can be computed with an uncertainty of about $2 \mathrm{~km} / \mathrm{s}$ (depending on which line is to be observed and which area of the SUMER detector is used). As the lines from the transition region and corona typically do show line shifts of several $\mathrm{km} / \mathrm{s}$ up to some 20 or $30 \mathrm{~km} / \mathrm{s}$ in the quiet Sun, SUMER is ideally suited for investigations of quiet Sun mass flows.

Very often the following terms are used synonymously, which one should not do as it is confusing the issue:

line shift - Doppler shift - Doppler velocity - mass flow.

Using each of these terms means implicit assumptions. The line shift is determined directly from the observed spectra (even though here we assume that at least the line core is, e.g., of single Gaussian shape). The physical cause might be a mass flow; however, there are many effects that can shift an emission line: e.g., compressible waves like sound waves, blends by other (unknown) emission lines or even absorption lines in one wing of the emission line profile (see Peter \& Judge 1999, Appendix B1). The effect of sound waves has been investigated already in the late 1960ies in the context stellar absorption lines (Eriksen \& Maltby 1967). Sound waves due to localized heat input by nanoflares in coronal loops have been proposed as the mechanism causing the persistent transition region redshifts (Hansteen 1993). A sound wave propagating towards an observer will cause a net blueshift if the spectra are averaged over one period or more. This is simply because of the relation of velocity and density fluctuations. Likewise a wave propagating away from the observer causes a redshift. This discussion is to emphasize that one has to be careful when interpreting line shifts as mass flows.

With this introduction in mind the following discussion will mainly deal with observations of quiet Sun line shifts and their interpretation. In most but not all cases the interpretation will include mass flows. If not stated otherwise the presented data were acquired using SUMER.

\section{Determination of absolute line shifts and the rest wavelength}

To determine the absolute line shift, i.e., the shift with respect to the rest wavelength, is complex and needs to be done with great care. Basically there have been three different techniques used for solar EUV spectroscopy:

1. Calibration lamp and rest wavelength. Here a calibration lamp is used for in-flight calibration of the wavelength axis. If all the wavelengths of the calibration lamp as well as the rest wavelength of the line of interest are known, the absolute shift of the line as observed on the Sun can be determined.

This method involves the potential danger that the calibration lamp might "get stuck" in the optical path. A lamp was successfully used in many missions.

2. Chromospheric lines and rest wavelength. Instead of a lamp one can also use chromospheric lines, e.g. of neutral atoms, as these lines show a line shift much smaller that the transition region and coronal lines that will be discussed here. (This is similar to terrestrial absorption lines often used in ground based solar spectroscopy).

3. Centre-to-limb variation of line shift. Assuming the shift of the line of interest is caused by predominantly vertical motions (mass flows or waves), the line shift should drop from disk centre to the limb following $\cos \vartheta$, with $\vartheta$ being 
the heliocentric angle. Statistically speaking, right at and above the limb the line shift should be zero for optically thin lines, as motions towards and away from the observer will cancel out. The major advantage is that this method is independent of the rest wavelength.

Method 3 is of special importance as many EUV lines, especially of highly ionised species, are only poorly known or not measured in the laboratory at all (e.g. $\mathrm{MgX}$ at $625 \AA$ ). Together with a calibration of the wavelength axis by chromospheric lines the rest wavelengths of e.g. Ne vIII (770 $\AA$ ) was determined to be $770.428 \pm 0.003 \AA$ (Peter \& Judge 1999, Dammasch et al. 1999) and that of $\mathrm{Mg} \mathrm{x}(625 \AA)$ to be $624.968 \pm 0.007 \AA$ (Peter \& Judge 1999).

Even though these considerations might seem boring at first sight, they are of vital importance for our interpretation of the data: The "new" values for the rest wavelength of Ne VIII and $\mathrm{MgX}$ differ by about $10 \mathrm{~km} / \mathrm{s}$ from the "old" values as listed e.g. in the standard line list of Kelly (1987). If using the "old" rest wavelengths both Ne VIII and $\mathrm{Mg} \mathrm{X}$ are redshifted at disk centre (e.g., Brekke et al. 1997, Chae et al. 1998). With the "new" rest wavelengths, however, both lines are blueshifted! And of course there is a major qualitative difference whether a line is blue- or redshifted. Thus these considerations are of vital importance for our understanding of the mass balance of the corona!

\section{Doppler shifts in coronal holes}

On a large scale coronal holes show a net blueshift - certainly in coronal lines, as shown already by Rottman et al. (1981), but there is also evidence for blueshifts in lines formed in the transition region. This is to be expected, as the fast solar wind is originating from the coronal holes. The connection of these line shifts to the magnetic field structure is still an active area of research.

The coronal hole as such. Lines from the low corona, like Ne VIII (770 $\AA$ ), are blueshifted in coronal holes (Warren et al. 1997). The blueshifts are present everywhere in the coronal hole (Peter 1999a) and the centre-to-limb variation within the hole closely follows a $\cos \vartheta$ law in good agreement with an (almost) radial outflow. From a $\cos \vartheta$ fit to the centre-to-limb variation an absolute value for the net outflow velocity can be derived with good accuracy to be about 12 $\mathrm{km} / \mathrm{s}$ (Peter 1999b). The average coronal hole line profile of Ne VIII is nonGaussian, probably with two equally strong Gaussian components (Wilhelm et al. 2000). This shows that the emission from the hole comes from (at least) two different structures.

The strong line of He I ( $584 \AA$ ) shows a net blueshift in coronal holes, too. However, the centre-to-limb variation of the line shift is not consistent with a radial flow and the line shows signs of self-absorption (Peter 1999b, Wilhelm et al. 2000). Thus it is unlikely that this is a signature of the solar wind.

The situation for lines formed in the transition region is less clear than for coronal lines. Using HRTS data (High Resolution Telescope and Spectrograph), Dere et al. (1989b) found for C IV (1548 $\AA$ ) a higher spatial fraction of blueshifts in a coronal hole than in the quiet Sun, but the line was still slightly redshifted on average. A similar result was obtained by Warren et al. (1997) for O IV. Peter \& Judge (1999) investigated a larger number of lines from the transition 
region, and found that the lines are generally blueshifted compared to quiet Sun emission, but still show a (small) net redshift. From these findings one can conclude that the solar wind outflow already sets in in the low transition region, but as a large part of the transition region emission in coronal holes is originating from small closed field regions, we do still see a net redshift.

A closer look at coronal hole blueshifts. The line shifts of Ne VIII $(770 \AA)$ in a coronal hole are anti-correlated to the line intensity in the sense that the strongest blueshifts occur at locations of lowest intensities (Wilhelm et al. 2000). This is in close agreement with theoretical arguments by Holzer et al. (1997). In magnetically open structures most of the energy flowing into the corona is used to accelerate the plasma and to form the solar wind. The more efficient the acceleration, the less energy is available for radiation. Thus one would expect the highest speeds to correspond to the lowest intensities.

The strongest blueshifts in Ne VIII are observed at intersections of network lanes, i.e., at locations where different network elements come together (Hassler et al. 1999). In a comparison with the magnetic field structure Xia et al. (2003) showed that these strongest blueshifts occur mainly where the magnetic field is unipolar down to $1-2^{\prime \prime}$ (resolution of the magnetograms). This supports the interpretation that at these network intersections the magnetic field forms funnel type structures in which the solar wind is efficiently accelerated (e.g. models of Marsch \& Tu 1997; Hackenberg et al. 2000).

Polar Plumes. The role of polar plumes as a contributor to the solar wind is still heavily debated. Determinations of elemental abundances in the plume show a strong enrichment of low-FIP elements (First Ionisation Potential) by a factor of up to 10 (Widing \& Feldman 1992). As this enrichment largely exceeds the FIP-fractionation in the fast solar wind (von Steiger et al. 2000) it was concluded that polar plumes cannot contribute significantly to the solar wind.

Wilhelm et al. (1998) found an outflow speed of about $18 \mathrm{~km} / \mathrm{s}$ in polar plumes close to the Sun. Using Doppler-dimming techniques with SUMER Patsourakkos \& Vial (2000) found outflow velocities of $\sim 70 \mathrm{~km} / \mathrm{s}$ at $0.05 R_{\odot}=$ $34 \mathrm{Mm}$ above the surface in an inter-plume region. Thus they concluded that plumes are not significant for the wind, though one has to note that this conclusion is not based on comparing plume and inter-plume within the same study using the same techniques.

More recently Gabriel et al. (2003) compared plume and inter-plume regions from 1.05 to $1.35 R_{\odot}$ in a more comprehensive study using Doppler dimming to derive the flow speed in both regimes. They find that at $1.1 R_{\odot}$ the plume and inter-plume regions contribute roughly equal amounts of mass to the solar wind. Thus the discussion on the nature of polar plumes is open again.

\section{Quiet Sun line shifts: observations}

If averaging line shifts over a large area of quiet Sun the median value of the shifts changes on the disk as $\cos \vartheta$ and is zero above the limb. Earlier data sets draw a confused picture simply because of their limitations in spatial coverage. 


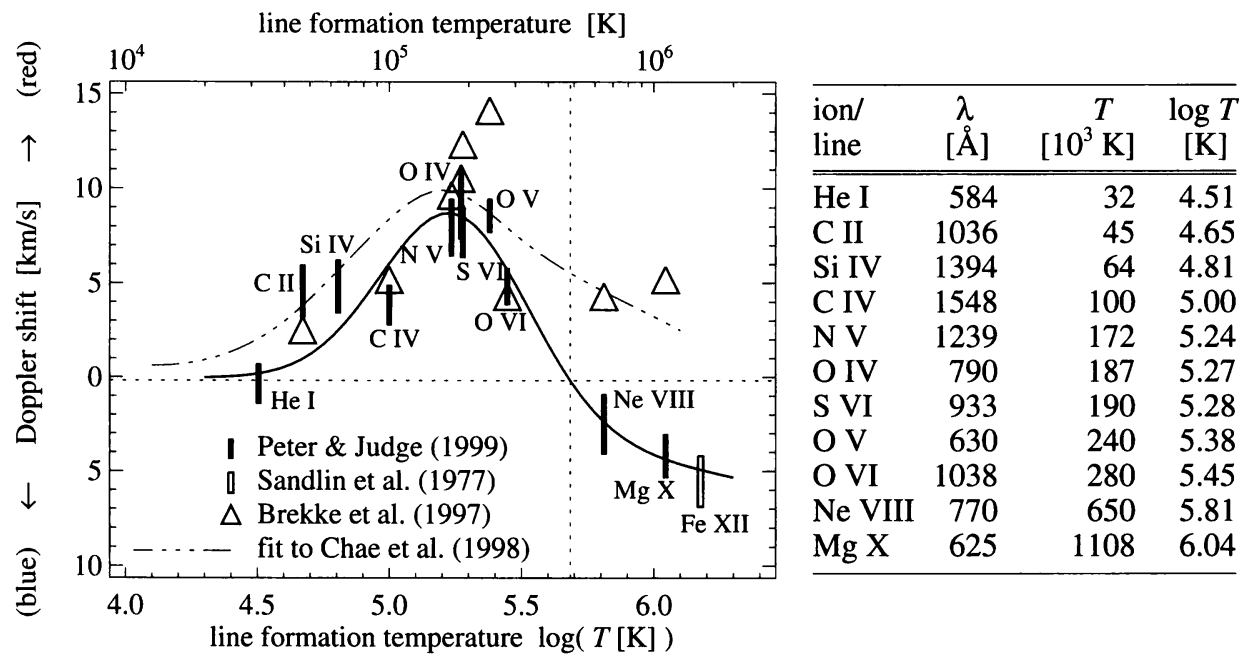

Figure 1. Line shifts at quiet Sun disk centre as a function of line formation temperature $T$. Values for $T$ and $\log T$ are listed in the right panel (taken from Landini \& Monsignori Fossi 1990).

With SUMER it could be shown that the $\cos \vartheta$ variation is a general property of the quiet Sun (Peter 1999a, Peter \& Judge 1999).

The average line shift on the disk increases with line formation temperature up to some $10-15 \mathrm{~km} / \mathrm{s}$ at $200000 \mathrm{~K}(\log T=5.3)$, then decreases again and turns into blueshifts above $500000 \mathrm{~K}$ (see Fig. 1). The situation is quite similar for (moderately) active regions (Teriaca et al. 1999a). This is a major constraint for any model of the transition region and low corona, because on average, i.e., in a statistical sense, this variation has to be reproduced.

Relation to the network structure. The line shifts are strongest in the supergranular network, as can be seen from scatter plots of intensity vs. Doppler shift (e.g. Peter 1999a). For lines from the middle transition region, especially for CIV, there is some evidence that the line shifts are non-zero in the network, while in the inter-network the line shifts are vanishing on average; for lines from the low corona there seems to be little relation of shifts and global network structure (e.g., Peter 1999a).

When investigated in more detail the very close connection of line shifts and photospheric magnetic field strength is clearly evident (e.g., Innes 2001), pointing out the close relation of Doppler shifts and magnetic activity. Results of Doyle et al. (2002) indicate that the line shifts are strongest at the edges of the bright network areas, i.e. at the boundaries from network to inter-network. Their study contains only a small number of cases, so more work is needed to substantiate this result.

The conclusion of (Hassler et al. 1999) for coronal holes holds also for quiet Sun regions, namely that the blueshifts of coronal lines are strongest at network intersections (cf. Sect. 3). 
Spatial variability. The line shifts as plotted in Fig. 1 are only average values. When observing a larger portion of the quiet Sun (a couple of network elements) with the highest possible spatial resolution, i.e., 1-2", the scatter of line shifts is much larger than the average value. The line shift distribution at disk centre is clearly non-symmetric (but very roughly Gaussian) with a full width at half maximum of $16 \mathrm{~km} / \mathrm{s}$ for C IV and $10 \mathrm{~km} / \mathrm{s}$ for Ne VIII. The distribution of shifts close to the limb is narrower by about $30 \%$, indicating that the motions causing the line shifts are indeed predominantly vertical (Peter 1999a, Fig. 9).

Temporal variability. The line shifts are also highly variable down to time scales of some $10 \mathrm{~s}$ and the variability is largest in the middle transition region. The scatter of the line shifts in time series is also very large (Hansteen et al. 2000) and comparable to the spatial variability.

At some locations a clear oscillatory variation of the line shifts with a 3 minutes period is observable, which is highly correlated with intensity variations indicating an upward propagating longitudinal compressible wave (Wikstøl et al. 2000). In general, the temporal RMS fluctuations of the Doppler shifts are highly correlated to the average Doppler shifts and to the RMS fluctuations of the relative intensity. This also supports a major role of compressible fluctuations (Brković et al. 2003).

\section{Understanding transition region line shifts (?)}

It is generally believed that the bulk part of the transition region emission is originating from magnetically closed structures, coronal loops. This seems very reasonable, as in magnetically open structures most of the energy flowing into the corona is used to accelerate the plasma and to form a solar wind (cf. Sect. 3). Models show that in the case of comparable energy input into open and closed field regions the emission from closed regions should dominate open regions by a factor of 10 (Holzer et al. 1997). Thus quiet Sun observations should basically show the closed field regions, i.e., loops.

\subsection{Single structure scenarios}

The first models to understand the transition region redshifts involved mass flows through loops. For example, if a syphon flow is driven by asymmetric heating, the flow might reach supersonic speed near the apex and form a shock in the downflowing leg. As the density in the shock region will be higher than in the upflowing leg, the redshifted emission will dominate - if the loop is not resolved by observations, only a redshift will be observed. Spadaro et al. (2000) have resolved some loops using SUMER spatial maps that support this picture. Many more scenarios and models have been developed in the 1980ies and 1990ies involving loop flows, too many to review them all here (see Peter \& Judge 1999 for a summary and Mariska 1992 for a more comprehensive review). The majority of these models considered stationary flows, which seems to contradict the observations that indicate a high temporal variability of emission and Doppler shifts mentioned above.

Furthermore none of these models is able to reproduce the observed turnover from red to blueshifts towards higher temperatures as shown in Fig. 1. Based on 


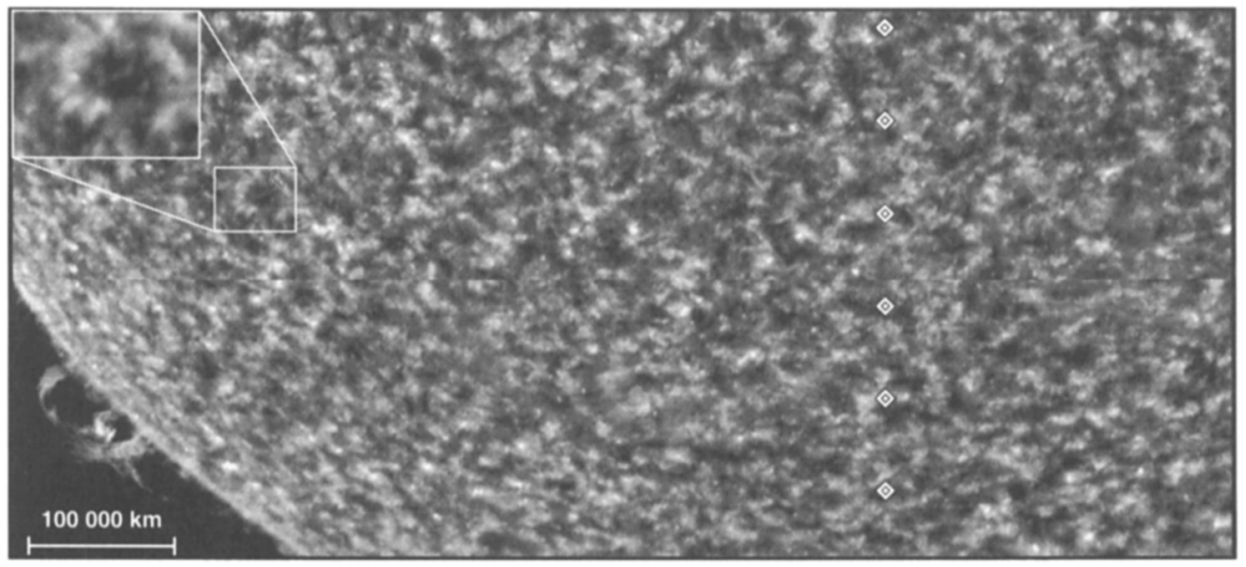

Figure 2. Image of the Sun in C III $(977 \AA)$ formed at $\sim 80000 \mathrm{~K}$ in the middle transition region. The diamonds show locations $100^{\prime \prime}, 200^{\prime \prime}$, $\ldots, 600^{\prime \prime}$ south of disc centre on the central meridian. A prominence can be seen above the limb. In the network small loops perpendicular to the network lanes are visible especially in the magnified inset.

the model of Hansteen (1993) this might be possible. In that model nanoflares were postulated to occur in the upper part of the loop. The very localised energy input leads to a localised increase of the pressure and by this generates sound waves propagating down the loop. These downward propagating waves will result in a net redshift of the emission lines (see Introduction) and naturally explain the highly dynamic nature. As the waves are propagating down the loops in both legs, one should see redshifts at both footpoints of the loop when observing at disc centre (and if the loops are perpendicular to the solar surface). Because the transition region emission is concentrated near the loop feet, one would see the strongest line shifts near the feet. If the loops are perpendicular to the network lanes, as suggested by Fig. 2, then the strongest redshifts should be observed not at the centre of the network patches, but at the boundaries from the network to the inter-network, as it is observed.

As the waves are reflected at the bottom of the loop one can also understand the large distribution of line shifts from blue to red for any given line. Peter \& Judge (1999) suggested that a modification of the Hansteen (1993) model, namely to relocate the preferred location of the nanoflares to lower temperatures, would also explain the net blueshifts for coronal lines and the turnover from red to blue in Fig. 1. Meanwhile this has been tested by Teriaca et al. (1999b), though their results agree only roughly qualitatively, but not quantitatively.

However, there is a major shortcoming of this latter scenario (and probably of every scenario trying to understand the line shifts using one single structure): What is the physical reason to assume that the nanoflares should occur just at around $500000 \mathrm{~K}$ ?

\subsection{Multiple structure scenarios}

Based on the morphology of the transition region emission, namely that the network is vanishing towards higher temperatures (Reeves 1976) a first model 
of the transition region based on funnels rooted in the super-granular network was developed by Gabriel (1976). However, enhanced transition region emission above small magnetic dipoles indicated the existence of small loops (Porter et al. 1987) and especially the lack of emission measure at low temperatures in Gabriel's model led Dowdy et al. (1986) to postulate a co-existence of coronal funnels and loops below and inbetween these funnels (see also Fig. 3). The existence of these small network loops seems quite clear from SUMER raster scans as shown in Fig. 2 (see also Feldman et al. 2003). Furthermode Landi et al. (2000) present evidence that the emission formed around $200000 \mathrm{~K}$ is dominated by cool loop-like structures.

Based on such a structure of the transition region one could postulate that the cooler loops (below $500000 \mathrm{~K}$ ) would produce the transition region redshifts in the same way as proposed by the early models (see above).

The blueshifts seen on average with coronal lines could then be attributed to a hotter class of loops. Though it would remain unclear why hot loops should produce blueshifts, while the cool loops produce redshifts.

An alternative scenario could be to postulate that funnels are the source of the observed blueshifts in hot lines. Then the blueshifts in the open funnels would be a signature of the solar wind outflow at the very base of the corona. This would then support the observation of the strongest blueshifts in coronal lines to be located at network intersections (Sect. 4). However, there is a major problem with this hypothesis. As mentioned earlier, open regions, i.e. the funnels, should be much fainter than closed field regions. So any closed field regions below or inbetween funnels reaching coronal temperatures would outshine the emission from the funnels. Thus it does not seen probable that the blueshifted emission from the coronal lines originates from funnels in the quiet Sun.

The situation in coronal holes, as discussed above, is quite different, as there one might safely assume that the open field regions dominate the emission.

\section{Funnels, loops and the transition region structure}

Let us take the suggestion on the structure of Dowdy et al. (1986) at face value, i.e., that loops and funnels do co-exist. Then the argument of Holzer et al. (1997), namely that funnels are fainter than loops, has a very interesting consequence. When observing from above, the line of sight will, generally, intersect funnels and well as loops. Then the spectral profiles should be composed from two components: a line core from the loops and a broader tail component from the funnels (the funnel emission will be less bright and because of the lower density in the funnel the lines will be more broadened by non-thermal effects, e.g. waves).

Exactly such double component spectra are observed in the network (firstly by Kjeldseth Moe \& Nicolas 1977, later by Dere \& Mason 1993). The tail component has three important properties (Peter 2000, 2001). (1) The tail component is present mostly (but not only) in the network: (2) The non-thermal broadening of the tail component increases monotonically with line formation temperature $\left(\propto T^{1 / 4}\right)$ and is about a factor of $2-3$ higher than for the line core and exceeds the sound speed. The increase with temperature is consistent with 


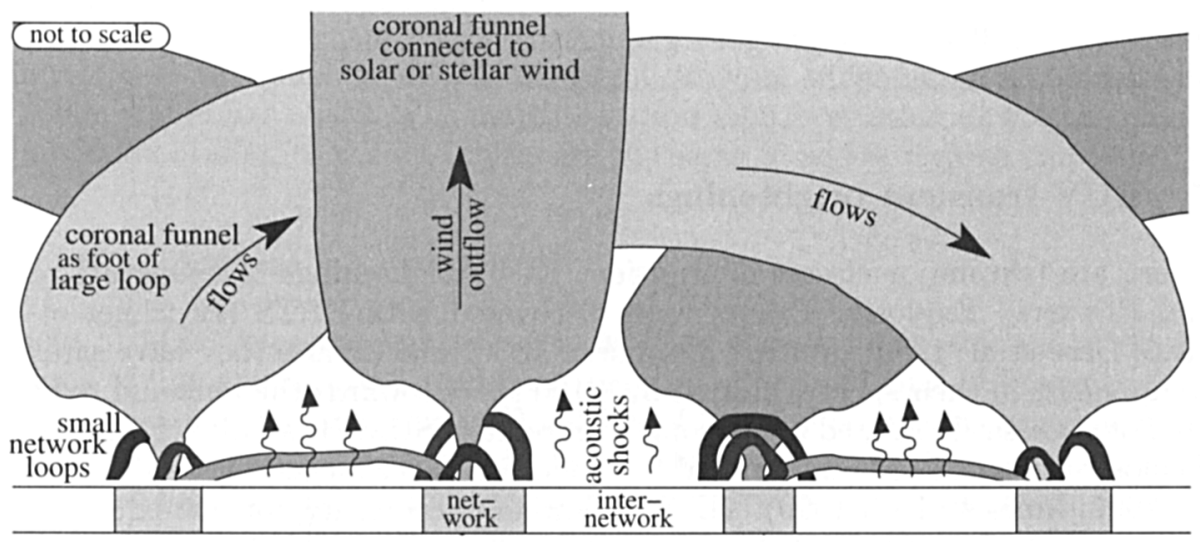

Figure 3. Sketch of the structure of the low corona.

an upward propagating Alfvén wave. (3) The tail components are systematically shifted towards the blue compared to the line core.

These observations would be consistent with coronal funnels coexisting with coronal loops. However, the emission of the funnels is not directly visible from the main part of the spectral profile, but one has to investigate the deviation of the line profile from a single Gaussian by applying a double Gaussian fit. The information from the coronal funnels is then contained in the broad tail component. The properties of the tail component are consistent with a plasma outflow powered by Alfvén wave heating. Thus one would end up with a scenario for the transition region structure a shown in Fig. 3.

One might argue that the tail components are not originating from coronal funnels, but are e.g. due to Explosive Events, i.e. small reconnection events causing bipolar flows (e.g., Dere et al. 1989a, Innes et al. 1997). If these are not very bright, they might indeed cause a line profile closely mimicking a double Gaussian (Chae et al. 2000). However, a recent study has shown that a triple Gaussian fit, as to be expected for an Explosive Event scenario, is less significant than a double Gaussian fit with a core and a tail component (Peter \& Brković 2003). This argues against a connection of Explosive Events and tail components.

This whole discussion on the interpretation of transition region line shifts shows a major shortcoming of existing (theoretical) work. The problem of interpreting the line shifts in terms of flows or waves is very closely connected to the assumptions made on the structure of the transition region. Of course, one can construct models for various structures and might find an agreement or disagreement with the observations. However, it is still unclear if the respective structure is really applicable to the solar corona, and if so, whether it is of any importance.

Therefore it is highly desirable to get a better understanding of the 3D structure of the transition region. Now first 3D MHD models for (small) parts of the corona become available (Gudiksen \& Nordlund 2002) and we might hope that further evolved models will allow a detailed comparison of models and 
observations. Then we might get a grip on the basic transition region structuring and decide on many of the above questions.

\section{EUV transient brightenings}

There are two major classes of transient EUV brightenings: Explosive Events and Blinkers. Explosive Events were discovered with HRTS (Bruckner et al. 1983; Dere et al. 1984). With a lifetime of about one minute they have satellite components in their spectra shifted by $\sim 100 \mathrm{~km} / \mathrm{s}$ towards the blue and red and are not necessarily related to a strong brightening. SUMER studies clarified that Explosive Events are bi-directional jets following a reconnection event (Innes et al. 1997; Innes \& Toth 1999). 2D MHD models accounting for non-equilibrium ionisation confirmed that picture (e.g. Roussev \& Galsgaard 2002).

EUV Blinkers were discovered by CDS on SOHO and are brightenings best observed in transition region lines which show an intensity increase of typically 60-80\% and last for about 5 to 30 minutes (Harrison et al. 1999; Brković et al. 2001; Bewsher et al. 2002). Priest at al. (2002) presented a number of blinker scenarios, one being that Blinkers might be due to a compression in the chromosphere, e.g. due to exploding granules. So far it is not clear whether or not, and if yes, how Blinkers and Explosive Events are related, even though many authors claim a connection (e.g., discussion in Peter \& Brković 2003).

At least one sub-class of EUV Blinkers might be related to strong flows through loops as has been shown in a recent SUMER investigation by Peter $\&$ Brković (2003). They present a case where the line shift increases by some $10 \mathrm{~km} / \mathrm{s}$ until the peak intensity is reached. As the structure extended some $4 \mathrm{Mm}$ along the slit, it could correspond to a loop with a footpoint distance of $4 \mathrm{Mm}$, i.e. a loop length of some $6 \mathrm{Mm}$. Interpreting the Doppler shift as a mass flow, the observed $10 \mathrm{~km} / \mathrm{s}$ correspond to a crossing time of about $10 \mathrm{~min}$ utes. This almost exactly matches the rise time of the intensity of the Blinker! Preliminary results of model calculations of a flow through a loop driven by asymmetric heating support this scenario.

\section{A short note on Active Regions}

The SUMER spectrometer was not used extensively for observations of active regions because of its limitations in photon counts. Of the few active region studies only one will be mentioned here.

Winebarger et al. (2002) studied line shifts of Ne VIII in an active region loop system, which consisted of fan-like structures seen by TRACE $171 \AA$ images (mainly Fe IX $/ \mathrm{X}$, i.e. at and above $10^{6} \mathrm{~K}$ ). They found high redshifts of up to $40 \mathrm{~km} / \mathrm{s}$ in Ne VIII $(770 \AA)$, a line formed at about $650000 \mathrm{~K}$ and interpreted these line shifts as being due to a syphon flow due to asymmetric heating. However, unlike in the study of Spadaro et al. (2000) they do not see both ends of the loops in the system, so their redshifts could also be draining material from the loop, having nothing to do with asymmetric heating and syphon flows at all.

The importance of this observation is that unlike on the quiet Sun and also unlike in the active region study by Terriaca et al. (1999a) they do not see small 
blueshifts, but strong redshifts in coronal lines. More complete studies have to investigate this further, as it is of special interest to identify the nature of the active region line shifts. The major question then is whether or not the physical processes leading to the line shifts are the same in active regions and quiet Sun.

\section{Conclusions}

The SUMER spectrograph presents us with an enormous amount of observations of line shifts in the transition region and corona that still awaits proper interpretation. So far $1 \mathrm{D}$ and $2 \mathrm{D}$ models of selected structures, e.g. loops, have been used widely to understand the line shifts. However, to draw final conclusions we have to understand the magnetic structure of the low corona, too, i.e. we have to investigate $3 \mathrm{D}$ coronal models. The spectroscopic results presented in this paper will provide hard boundary conditions to test such future models.

\section{References}

Bewsher, D., Parnell, C.E., \& Harrison, R.A. 2002, Solar Phys., 206, 21

Brekke, P., Hassler, D.M., \& Wilhelm, K. 1997, Solar Phys., 175, 349

Brković, A., Solanki, S.K., \& Rüedi, I. 2001, A\&A, 373, 1056

Brković, A., Peter, H., \& Solanki, S.K. 2003, A\&A, 403, 725

Brueckner, G.E., \& Bartoe, J.-D.F. 1983, ApJ, 272, 329

Chae, J., Yun, H.S., \& Poland, A.I. 1998, ApJS, 114, 151

Chae, J., Wang, H., Goode, P.R., et al. 2000, ApJ, 528, L119

Dammasch, I.E., Wilhelm, K., Curdt, W., \& Hassler, D. 1999, A\&A, 346, 285

Dere, K.P., Bartoe, J.-D.F., \& Brueckner, G.E. 1984, ApJ, 281, 870

Dere, K.P., Bartoe, J.-D.F., \& Brueckner, G.E. 1989a, Solar Phys., 123, 41

Dere, K.P., Bartoe, J.-D.F., Brueckner, G.E., \& Recely, F. 1989b, ApJ, 345, L95

Dere, K.P., \& Mason, H.E. 1993, Solar Phys., 144, 217

Doshek, G.A., Feldman, U., \& Bohlin, J.D. 1976, ApJ, 205, L177

Dowdy, J.F., Rabin, D., \& Moore, R.L. 1986, Solar Phys., 105,

Doyle, J.G., Madjarska, M.S., Roussev, I., et al. 2002, A\&A, 396, 255

Eriksen, G., \& Maltby, P. 1967, ApJ, 148, 833

Feldman, U., Dammasch, I.E, Wilhelm, K., et al. 2003, Images of the solar upper atmosphere from SUMER on SOHO, ESA SP-1274.

Fleck, B., Domingo, V., \& Poland, A. I., eds. 1996, The SOHO mission (Solar Physics Vol. 162), Kluwer Academic Publishers, Dordrecht

Gabriel, A.H. 1976, Phil. Trans. Roy. Soc. Lond., A281, 339

Gabriel, A.H., Bely-Dubau, F., \& Lemaire, P. 2003, ApJ, 589, 623

Gudiksen, B.V., \& Nordlund, Å., 2002, ApJ, 572, L113

Hackenberg, P., Marsch, E., \& Mann, G. 2000, A\&A, 360, 1139

Hansteen, V.H. 1993, ApJ, 402, 741

Hansteen, V.H., Betta, R., \& Carlsson, M. 2000, A\&A, 360, 742

Harrison, R.A., Lang, J., Brooks, D.H., \& Innes, D.E. 1999, A\&A, 351, 1115 
Hassler, D.M., Dammasch, I.E., Lemaire, P. et al. 1999, Science 283, 810

Holzer, T.E., Hansteen, V.H., \& Leer, E. 1997, in Cosmic winds and the heliosphere, eds. J.R. Jokipii, C.P. Sonett, \& M.S. Giampapa (Univ. of Arizona Press, Tucson), 239

Innes, D. 2001, A\&A, 378, 1067

Innes, D., Inhester, B., Axford, W.I., \& Wilheln, K., 1997, Nature, 386, 811

Innes, D.E., \& Tóth, G. 1999, Solar Phys., 185, 127

Kelly, R. L. 1987, J. Phys. Chem. Ref. Data Suppl., 16, 1

Krieger, A.S., Timothy, A.F., \& Roelof, E.C. 1973, Solar Phys., 23,123

Kjeldseth Moe, O., \& Nicolas, K.R. 1977, ApJ, 211, 579

Landi, E., Mason, H.E., Lemaire, P., \& Landini, M., A\&A, 357, 743

Landini, M., \& Monsignori Fossi, B.C. 1990, A\&AS, 82, 229

Lemaire, P., Wilhelm, K., Curdt, W., et al. 1997, Solar Phys., 170, 105

Mariska, J.T. 1992, The Solar Transition Region, (Cambridge Univ. Press, Cambridge)

Marsch, E., \& Tu, C.-Y., 1997, Solar Phys., 176, 87

Patsourakkos, S., \& Vial, J.-C. 2000, A\&A, 359, L1

Peter, H. 1999a, ApJ, 516, 490

Peter, H. 1999b, ApJ, 522, L77

Peter, H. 2000, A\&A, 360, 761

Peter, H. 2001, A\&A, 374, 1120

Peter, H., \& Brcović, A. 2003, A\&A, 403, 287

Peter, H., \& Judge, P.G. 1999, ApJ, 522, 1148

Porter, J.G., Moore, R.L., Reichmann, E.J., et al. 1987, ApJ, 323, 380

Priest, E.R., Hood, A.W., \& Bewsher, D. 2002, Solar Phys., 205, 249

Sandlin, G., Brueckner, G.E., \& Tousey, R. 1977, ApJ, 214, 898

Spadaro, D., Lanzafame, A.C., Consoli, L., et al. 2000, A\&A, 359, 716

Reeves, E.M. 1976, Solar Phys., 46, 53

Rottman, G.J., Orrall, F.Q., \& Klimchuk, J.A. 1981, ApJ, 247, L135

Roussev, I., \& Galsgaard, K. 2002, A\&A, 383, 697

Teriaca, L., Banerjee, D. \& Doyle, J.G. 1999a, A\&A, 349, 636

Teriaca, L., Doyle, J.G., Erdélyi, R., \& Sarro, L.M. 1999b, A\&A, 352, L99

von Steiger, R., Schwadron, N.A., Fisk, L.A., et al. 2000, J.G. R., 105, 27217

Warren, H., Mariska, J., \& Wilhelm, K. 1997, ApJ, 490, L187

Widing, K.G., \& Feldman, U. 1992, ApJ, 392, 715

Wilhelm, K., Curdt, W., Marsch, E., et al. 1995, Solar Phys., 162, 189

Wilhelm, K., Lemaire, P., Curdt, W., et al. 1997, Solar Phys., 170, 75

Wilhelm, K., Marsch, E., Dwivedi, B.N., et al. 1998, ApJ, 500, 1023

Winebarger, A.R., Warren, H., van Ballegooijen, A., et al. 2002, ApJ, 567, L89

Wikstøl, Ø., Hansteen, V.H., Carlsson, M., \& Judge, P.G. 2000 ApJ, 531, 1150

Xia, L.D., Marsch, E., \& Curdt, W. 2003, A\&A, 399, L5 